\title{
Multiferroicity and colossal magneto-capacitance in Cr-thiospinels
}

\author{
J. Hemberger ${ }^{1}$, P. Lunkenheimer ${ }^{1}$, R. Fichtt ${ }^{1}$, S. Weber ${ }^{1}$, V. Tsurkan ${ }^{1,2}$, and A. Loidl ${ }^{1}$ \\ ${ }^{1}$ Experimental Physics V, Center for Electronic Correlations and Magnetism, \\ University of Augsburg, D-86135 Augsburg, Germany \\ ${ }^{2}$ Institute of Applied Physics, Academy of Sciences of Moldova, Chisinau, Republic of Moldova
}

\begin{abstract}
The sulfur based Cr-spinels $R \mathrm{Cr}_{2} \mathrm{~S}_{4}$ with $R=\mathrm{Cd}$ and $\mathrm{Hg}$ exhibit the coexistence of ferromagnetic and ferroelectric properties together with a pronounced magnetocapacitive coupling. While in $\mathrm{CdCr}_{2} \mathrm{~S}_{4}$ purely ferromagnetic order is established, in $\mathrm{HgCr}_{2} \mathrm{~S}_{4}$ a bond-frustrated magnetic ground state is realized, which, however, easily can be driven towards a ferromagnetic configuration in weak magnetic fields. This paper shall review our recent investigation for both compounds. Besides the characterization of the magnetic properties, the complex dielectric permittivity was studied by means of broadband dielectric spectroscopy as well as measurements of polarization hysteresis and pyro-currents. The observed colossal magneto-capacitive effect at the magnetic transition seems to be driven by an enormous variation of the relaxation dynamics.

PACS numbers: $75.80 .+q ; 77.22 . \mathrm{Gm}$
\end{abstract}

\section{INTRODUCTION}

In recent years multiferroic magnetoelectrics attracted increasing scientific and technological interest. ${ }^{1}$ In this rare class of compounds, ferroelectricity (or at least a weak ferroelectric component) and (ferro-)magnetism coexist and both order-parameters are strongly coupled. This coupling of the dielectric and magnetic properties makes them highly attractive not only from an academic point of view, but also for potential applications in microelectronics. Ferromagnetism is known since ancient times and a prerequisite of modern technology in many areas. Ferroelectricity has been identified in 1921 by Valasek $^{2}$ in Seignette salt and the existence of ferroelectricity in an oxide has been established as late as 1943 in $\mathrm{BaTiO}_{3}$ by von Hippel and coworkers. ${ }^{3}$ Ferroelectrics play an important role in thze technological revolution in optics, acoustics, and capacitor engineering for a variety of applications in electronic circuitry and modern energy storage. The coexistence of ferroelectricity and ferromagnetism would constitute another mile stone for modern electronics and functionalized materials. The most appealing applications are new types of storage media using both magnetic and electric polarization and the possibility of electrically reading/writing magnetic memory devices (and vice versa). The first multiferroic material, revealing magnetic and electric order to be discovered was nickel-iodine boracite in $1966^{4}$ and nowadays multiferroic materials are in the focus of interest of recent solid state research. Prominent examples for such type of materials are the heavy rare earth manganites like the perovskites $\mathrm{TbMnO}_{3}$ or $\mathrm{DyMnO}_{3},{ }^{5,6}$ orthorombic $\mathrm{TbMn}_{2} \mathrm{O}_{5},{ }^{7}$ the hexagonal $\mathrm{YMnO}_{3}$ and $\mathrm{HoMnO}_{3},{ }^{8,9}$ or the kagomé staircase compound $\mathrm{Ni}_{3} \mathrm{~V}_{2} \mathrm{O}_{8} \cdot{ }^{10}$ This contribution summarizes the results on the recently discovered multiferroic and magneto-capacitive properties of the cubic spinel systems $\mathrm{CdCr}_{2} \mathrm{~S}_{4}$ and $\mathrm{HgCr}_{2} \mathrm{~S}_{4} \cdot{ }^{11,12}$ We report on magnetization, dielectric polarization, as well as broadband dielectric measurements in these systems and provide detailed information on the dielectric relaxation dynamics. In addition, the influence of impurity doping and sample dependence is addressed. Spinel compounds are an important class of materials: For example, spinels are a key constituent in the deep-Earth mantle; and they are great imposters in gemstone history, often mistaken for rubies, even in crown jewels. The magnetic properties of spinel-type compounds have long been known and are in the focus of applied research since more than 50 years. Ferrites with spinel structure are being used for many applications in microwave technology and high-frequency electronics. Spinels have also been in the focus of basic research. The metal-insulator transition in $\mathrm{Fe}_{3} \mathrm{O}_{4}$ was described by Vervey ${ }^{13}$ in 1939 as a charge-ordering transition but this interpretation remains controversial. Recent reports on geometrical frustration of the spin and orbital degrees of freedom, ${ }^{14}$ and the observation of an orbital glass state ${ }^{15}$ in sulpho spinels, demonstrate the rich and complex physics, characteristic of these compounds. The richness is a result of the cooperativity of and the delicate balance between charge, spin, orbital, and lattice degrees of freedom.

At room temperature $\mathrm{CdCr}_{2} \mathrm{~S}_{4}$ and $\mathrm{HgCr}_{2} \mathrm{~S}_{4}$ are normal cubic $A B_{2} \mathrm{~S}_{4}$ thio-spinel compounds. ${ }^{11}$ The $\mathrm{Cd}^{2+}$ or $\mathrm{Hg}^{2+}$ ions exhibit an electronic configuration of filled $d^{10}$ shells. Thus on the structural $A$-sites, which form a diamond lattice, there are no magnetic or orbital degrees of freedom. The $\mathrm{Cr}^{3+}$ ions on the octahedrally coordinated $B$-sites, which form a pyrochlore lattice, possess half filled $t_{2 g}$ shells and thus also are orbitally inactive. But due to the quenched orbital moment and Hund's coupling, the $B$-sites carry a $S=J=3 / 2$ spin configuration. This simplifies in general the more complex orbital and magnetic interaction scenarios which can be found in this structural class of materials, as illustrated, e.g., by the case of $\mathrm{Fe}$ on the $A$-sites. ${ }^{14,16}$ The absence of orbital degrees of freedom in an highly symmetric lattice, changes the prerequisites for polar order in the compound. Jahn-Teller active orbital degrees of freedom may trigger non-polar lattice distortions. Then the $\mathrm{cu}-$ bic lattice relaxes into a Jahn-Teller distorted, orbitally 
ordered structure of lower symmetry. These distortions can be expected to be non-polar due to the symmetry of the electronic charge distribution of the orbital, to which the positions of the surrounding ligands are adapted. ${ }^{17}$ An example is the Jahn-Teller active system $\mathrm{FeCr}_{2} \mathrm{~S}_{4} \cdot{ }^{15}$ In contrast, the absence of any orbital degrees of freedom, as it is realized via the $d^{0}$ electronic configuration in canonical ferroelectrics like $\mathrm{BaTiO} 3$, allows for the relaxation of the structure into polar off-center distortions. In $\mathrm{CdCr}_{2} \mathrm{~S}_{4}$ and $\mathrm{HgCr}_{2} \mathrm{~S}_{4}$ the relatively large radii of the $A$-site ions destabilize the cubic structure even more. Accordingly, the tendency towards ionic off-center distortions is reported in literature for a number of spinel compounds. ${ }^{18} \mathrm{In}$ addition, for $\mathrm{CdCr}_{2} \mathrm{~S}_{4}$ negative thermal expansion and the anomalous broadening of x-ray Braggreflections was detected, denoting the softening of the spinel structure towards lower temperatures. ${ }^{19}$ Concerning the spin sector, in $\mathrm{CdCr}_{2} \mathrm{~S}_{4} \mathrm{Cr}$-spins, located on the $B$-site pyrochlore lattice, are coupled ferromagnetically, yielding a transition temperature of $T_{c}=84 \mathrm{~K}$. The situation is more complex in $\mathrm{HgCr}_{2} \mathrm{~S}_{4}$, which is close to ferromagnetism, but where the influence of bond-frustration results in the suppression of the ferromagnetic transition in zero magnetic field. ${ }^{20}$ Instead, a spiral-like antiferromagnetic spin structure is established below $T_{N}=22 \mathrm{~K}$. However, the antiferromagnetic ground state can be overcome already by weak external magnetic fields and ferromagnetism can be induced. In addition, even in the absence of an external field, at higher temperatures evidence for strong ferromagnetic fluctuations was found. ${ }^{20}$

\section{EXPERIMENTAL DETAILS}

The single crystals were grown from the polycrystalline starting material by chemical transport with chlorine as transport agent. The growth experiments were performed at temperatures between 800 and $850{ }^{\circ} \mathrm{C}$. Poly-crystalline $\mathrm{HgCr}_{2} \mathrm{~S}_{4}$ and $\mathrm{CdCr}_{2} \mathrm{~S}_{4}$ samples were prepared by conventional solid-state reaction in evacuated quartz ampoules from high purity (99.999\%) binary mercury sulfide and elementary $\mathrm{Cr}$ and $\mathrm{S}$. In order to reach good homogeneity, the compounds were grinded and resynthesized several times. The final tempering was performed at $800{ }^{\circ} \mathrm{C}$ in an atmosphere of sulfur excess. In the following, all data refer to single-crystalline samples if nothing contrary is explicitly mentioned. All batches were characterized by powder x-ray diffraction and found to exhibit the normal cubic spinel structure with space group $F d \overline{3} m$ without any indications of impurity phases. The magnetization measurements were performed with a commercial SQUID magnetometer (MPMS, QuantumDesign) in fields up to $5 \mathrm{~T}$. For the dielectric measurements, sputtered gold- or silverpaint electrodes were applied on opposite sides of the plate-like samples. The complex permittivity (dielectric constant and loss) was determined over a broad frequency range $(3 \mathrm{~Hz}<\nu<3 \mathrm{GHz})$ using frequency- response analysis and reflectometric techniques. ${ }^{21}$ The electric polarization was detected directly, employing a high-impedance Sawyer-Tower circuit, or via integration of the pyro-current measured with an electrometer. ${ }^{22} \mathrm{~A}$ conventional ${ }^{4} \mathrm{He}-$ bath cryostat and a commercial cryomagnet (Teslatron, OxFORD) allowed measurements at temperatures down to $1.5 \mathrm{~K}$ and in magnetic fields up to $10 \mathrm{~T}$.

\section{RESULTS AND DISCUSSION}

\section{A. $\mathrm{CdCr}_{2} \mathrm{~S}_{4}$}

Fig. 1 compares the dielectric and magnetic behavior of $\mathrm{CdCr}_{2} \mathrm{~S}_{4}$. The upper frame (Fig. 1a, right scale) shows the inverse magnetic susceptibility. A typical linear Curie-Weiss type behavior can be detected at elevated temperatures. The corresponding fitting values are a Curie-Weiss temperature of roughly $T_{C W} \approx 150 \mathrm{~K}$ and an effective paramagnetic moment close to the expected value of $p_{\text {eff }}=3.87 \mu_{B}$ per $\mathrm{Cr}^{3+}$. Already well above the ferromagnetic transition at $T_{c} \approx 84 \mathrm{~K}$, clear deviations from the linear behavior are observed due to the onset of fluctuations. In this regime the inverse susceptibility can be described as $\chi^{-1} \propto\left(T-T_{c}\right)^{\gamma}$ where the exponent $\gamma=1.41$ is close to the value for the $3 \mathrm{~d}$-Heisenberg model of 1.39 . Below $T_{c} \approx 84 \mathrm{~K}$ spontaneous magnetization sets

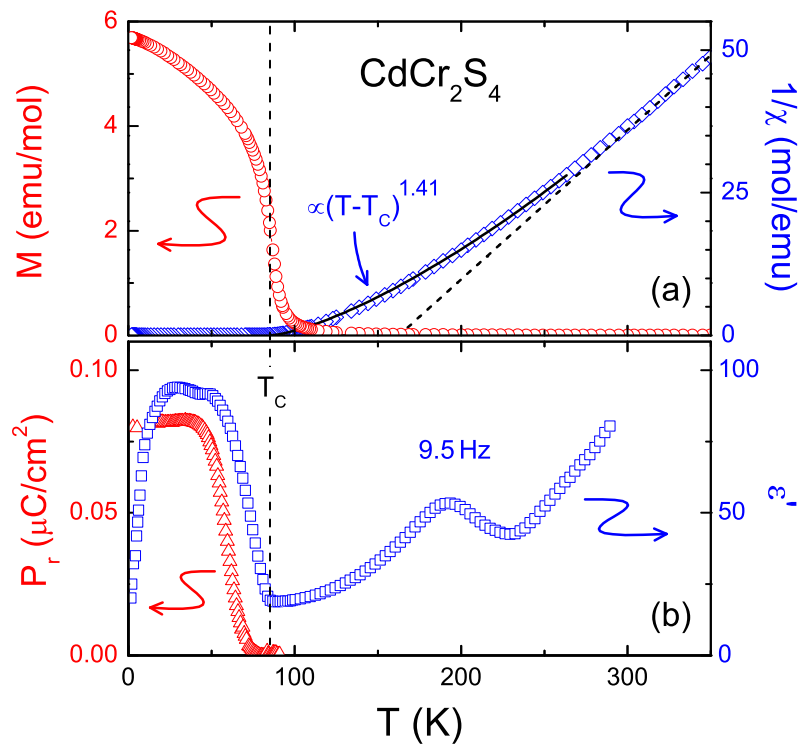

FIG. 1: Comparison of dielectric and magnetic properties in $\mathrm{CdCr}_{2} \mathrm{~S}_{4} \cdot{ }^{11}$ (a): Inverse magnetic susceptibility (right scale) and low field magnetization (left scale) vs. temperature measured at field of $H=1 \mathrm{kOe}$. (b): Dielectric permittivity at $9.5 \mathrm{~Hz}$ (right scale) and remnant polarization (left scale) vs. temperature. The polarization was obtained via integration of the pyro-current as measured on heating after poling in an electric field of $50 \mathrm{kV} / \mathrm{m}$. 


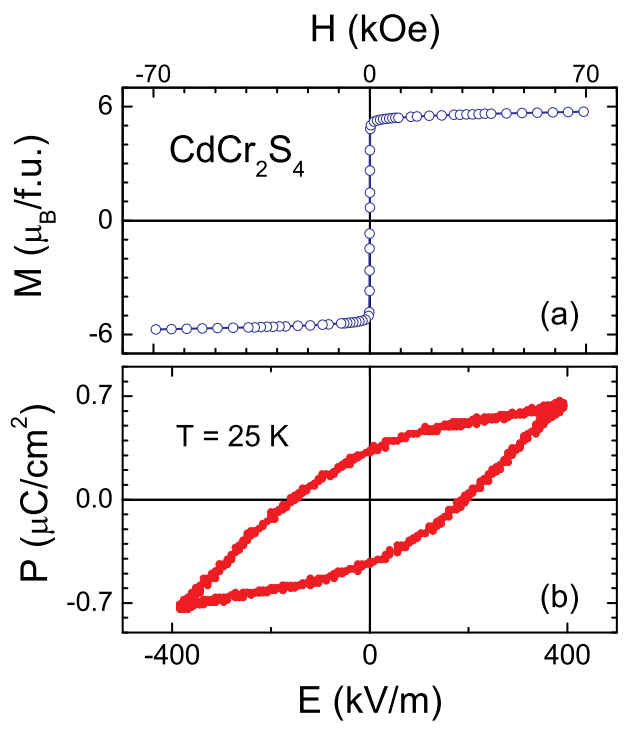

FIG. 2: Hysteresis loops for the magnetic (a) and dielectric sector (b) measured at $T=25 \mathrm{~K}$. The magnetic data measured at $H>0$ was mirrored and corrected for demagnetization effects; the polarization data was measured at $\nu=1.13 \mathrm{~Hz}$. (taken from [23])

in (left scale of Fig. 1)a). At the same time a steep increase of the dielectric constant can be detected, which for low frequencies reaches relatively high values above $\varepsilon^{\prime} \approx 100$ (Fig. 1b, right scale). The details of the frequency and temperature dependence of the complex permittivity in the paramagnetic regime will be discussed below. As shown in Fig. 1b (left scale), the low temperature phase of $\mathrm{CdCr}_{2} \mathrm{~S}_{4}$ in addition to spontaneous magnetization exhibits remnant polarization, which sets in shortly below $T_{c}$. Thus the high values of $\varepsilon^{\prime}$ can be interpreted as precursor of the onset of remnant dielectric polarization at lower temperatures. Fig. 2 shows magnetic (a) and dielectric (b) hysteresis loops measured at $25 \mathrm{~K} . M(H)$ exhibits the characteristics of a soft ferromagnet reaching nearly the full magnetic moment of $6 \mu_{B}$ expected for two $\mathrm{Cr}^{3+}$ ions per formula unit. The $P(E)$ experiment reveals a well pronounced hysteresis loop. Complete saturation is not reached within the experimentally accessible electric field range of $400 \mathrm{kV} / \mathrm{m}$ and no well defined coercive electric field strength can be identified. Such type of hysteresis behavior is typical for relaxor-ferroelectric behavior, where nano-scale ferroelectric clusters determine the polarization response ${ }^{24}$.

The relaxor-ferroelectric type of behavior shows up also in the the frequency and temperature dependence of the complex permittivity. Fig. 3 displays dielectric constant $\varepsilon^{\prime}(T)$ and the conductivity $\sigma^{\prime}(T)$ for frequencies from $3 \mathrm{mHz}$ to $3 \mathrm{GHz}$. In the paramagnetic regime a peak in $\varepsilon^{\prime}(T)$ shows up. This feature shifts to lower temperatures and increases in amplitude with decreasing frequency. The dashed line indicates a Curie-Weiss law, $\propto 1 /(T-130 \mathrm{~K})$, for the right flank of the peaks,

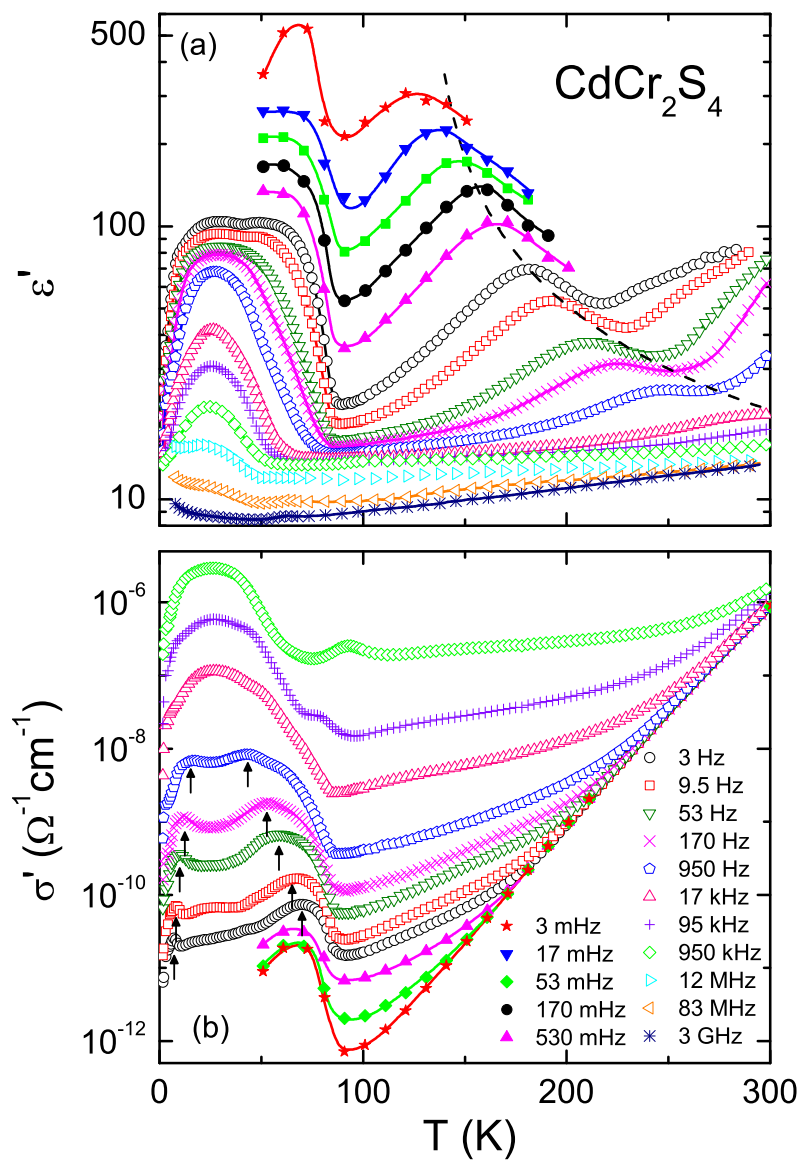

FIG. 3: Temperature dependence of the real part of the dielectric permittivity $\varepsilon^{\prime}$ (upper frame) and the ac-conductivity $\sigma^{\prime} \propto \omega \varepsilon^{\prime \prime}$ (lower frame) of $\mathrm{CdCr}_{2} \mathrm{~S}_{4}$ for various frequencies. The arrows indicate the occurrence of two maxima in $\sigma^{\prime}(T)$. At the magnetic transition $T \approx 84 \mathrm{~K}$ both $\varepsilon^{\prime}$ and $\sigma^{\prime}(T)$ exhibit an increase towards low temperatures ([25]; copyright (2005) by the American Physical Society).

which can be taken as an estimate of the static dielectric constant. The further increase of $\varepsilon^{\prime}(T)$ towards higher temperatures is due to contact and conductivity contributions as discussed below. However, the most characteristic feature in the temperature regime above $100 \mathrm{~K}$ is the described relaxational maximum of the dielectric constant. This type of behavior in $\mathrm{CdCr}_{2} \mathrm{~S}_{4}$ resembles that observed in relaxor ferroelectrics. ${ }^{24,26}$ In such compounds, the reduction of $\varepsilon^{\prime}$ below the peak temperature is usually ascribed to a cooperative freezing of ferroelectric clusters on the time scale given by the frequency of the applied AC electric-field. This is in contrast to canonical ferroelectrics where the frequency dependence of $\varepsilon^{\prime}(T)$ in the sub-GHz regime is negligible, even in the vicinity of the phase transition. The Arrhenius-type thermal activation of this relaxation process can be characterized by an energy barrier $E_{B} \approx 330 \mathrm{meV}$ and a high-temperature relaxation time $\tau_{0} \approx 1.1 \times 10^{-11} \mathrm{~s}$. The latter value is relatively small compared to canonical relaxors,${ }^{24,26}$ which 
may point to a small size of relaxing clusters. The relaxational features seen in the real part of the permittivity according to the Kramer-Kronig relation should be accompanied by peaks in the imagninary part located close to the points of inflection at the left wing of the maxima in $\varepsilon^{\prime}(T)$. However, in Fig. 3b, where $\sigma^{\prime}=\omega \varepsilon_{0} \varepsilon^{\prime \prime}$ ( $\varepsilon_{0}$ is the permittivity of the vacuum) is displayed, no such peaks become obvious. (As the temperature dependence of $\varepsilon^{\prime \prime}$ and $\sigma^{\prime}$ is identical (except for the absolute values), we chose to plot the latter to avoid a crossing of the curves and to enhance the readability of the figure.) This is due to the superimposition by contributions from charge carrier transport. Above $100 \mathrm{~K}$ the data of $\sigma^{\prime}(T)$, measured at the lowest displayed frequency of $3 \mathrm{mHz}$, represents the static DC conductivity. The levelling-off of the higher frequency data on decreasing temperature can be understood as AC-conductivity, usually determined by incoherent charge transport, i.e. hopping processes. The AC-conductivity usually increases with increasing frequency, which leads to the staggered behavior of the $\sigma^{\prime}$ curves for different frequencies. These additional conductivity contributions show up in the dielectric loss as $\varepsilon_{A C}^{\prime \prime}=\sigma_{A C} /\left(\omega \varepsilon_{0}\right)$ and make it difficult to evaluate the relaxational contributions to the dielectric loss. ${ }^{11,25}$

The most remarkable feature in Fig. 3 is the strong increase of $\varepsilon^{\prime}(T)$ below the ferromagnetic transition temperature $T_{c}=84 \mathrm{~K}$. It indicates the close coupling of magnetic and dielectric properties. This behavior can be eyplaine assuming that the frozen-in dielectric dynamics becomes fast again at the magnetic transition, thus restoring the large contribution to the dielectric response of the relaxing entities. ${ }^{25}$ The dielectric loss monitored via $\sigma^{\prime \prime}$ also increases below $T_{c}$. As mentioned before, this in part may be influenced by changes in the conductivity. Magnetoresistive effects have been reported for $\mathrm{CdCr}_{2} \mathrm{~S}_{4}$ and $\mathrm{CdCr}_{2} \mathrm{Se}_{4}$ in literature ${ }^{27}$. However, at least for the lower frequencies in Fig. 3b there is a two-peak structure, which can be detected within the ferromagnetic regime. The high-temperature peaks can be interpreted as the loss maxima connected to the re-acceleration of the relaxation dynmics below $T_{c}$. The low temperature peaks mirror the final freezing at lowest temperatures. The complete temperature dependence of the intrinsic relaxation time shall be discussed later and is displayed together with that of $\mathrm{HgCr}_{2} \mathrm{~S}_{4}$ in Fig. 11.

The changes of the relaxation dynamics and the corresponding increase of the dielectric response below the ferromagnetic transition are related to the onset of the magnetic order parameter, the ferromagnetic magnetization, and thus can also be influenced by an external magnetic field. Fig. 4a shows data of the dielectric constant for two different frequencies in zero external field and in $5 \mathrm{~T}$. The onset of the rise in $\varepsilon^{\prime}(T)$ is shifted to higher temperatures and smeared out upon the influence of the magnetic field according to the expected behavior of the magnetization. This results in a strong magnetic field dependence of $\varepsilon^{\prime}$ even well above the spontaneous magnetic transition temperature, which is documented

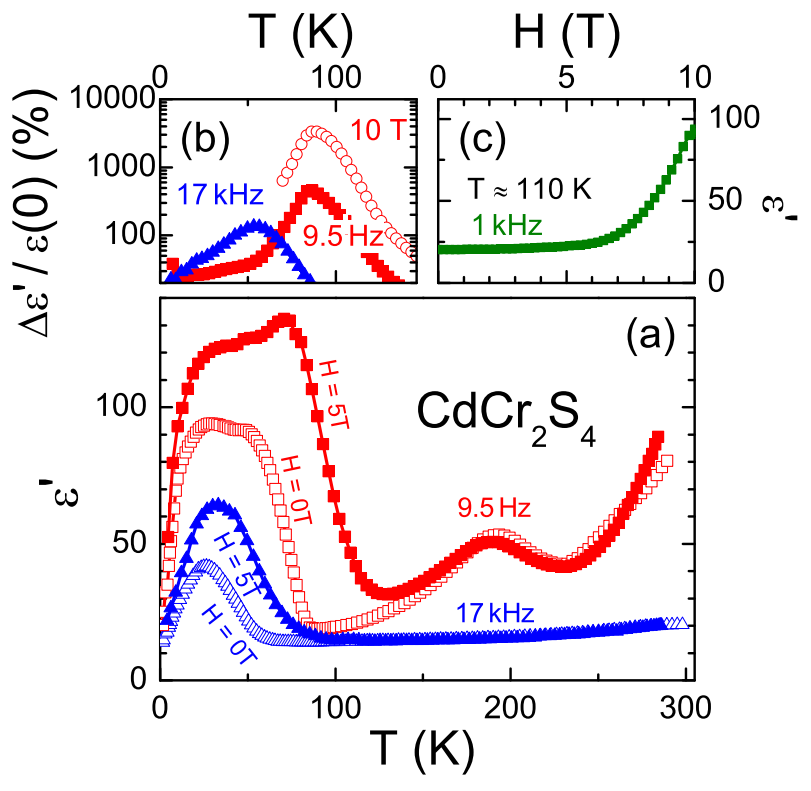

FIG. 4: Dielectric constant $\varepsilon^{\prime}(T)$ for frequencies of $9.5 \mathrm{~Hz}$ and $17 \mathrm{kHz}$ and magnetic fields of zero and $5 \mathrm{~T}$ (a). ${ }^{11,25}$ Frame (b): The temperature dependence of the magneto-permittivity as measured at $5 \mathrm{~T}$ (closed symbols) for $9.5 \mathrm{~Hz}$ and $17 \mathrm{kHz}$, and at $10 \mathrm{~T}$ (o) for $9.5 \mathrm{~Hz}$ on a logarithmic scale. In (c) the magnetic field dependence at $T=110 \mathrm{~K}$ is shown.

in Fig. 4c. Defining the magneto-capacitance as the ratio of the magnetic-field induced change of the capacity and the zero-field value, one arrives at values of up to $500 \%$ in a field of $5 \mathrm{~T}$ and up to $3000 \%$ in a field of $10 \mathrm{~T}^{25}$ as displayed in in Fig. 4b. In analogy to the similar scenario of a magnetic-field induced shift of a magnetic transition, causing the occurrence of colossal magnetoresisitive effects (CMR), e.g. in manganites, one may note the above described behavior in $\mathrm{CdCr}_{2} \mathrm{~S}_{4}$ as colossal magneto-capacitive effect (CMC). However, this effect appears to be strongly frequency dependent as it seems to be intimately coupled to the dynamics of the ferroelectric relaxor entities dominating the dielectric response.

\section{B. Doped and poly-crystalline $\mathrm{CdCr}_{2} \mathrm{~S}_{4}$}

The results shown so far have been obtained from samples of single-crystalline $\mathrm{CdCr}_{2} \mathrm{~S}_{4}$. In the following, we want to address the sample dependence of the dielectric properties by providing results on doped and polycrystalline material. Fig. 5 displays the real parts of the dielectric permittivity and AC-conductivity of $\mathrm{CdCr}_{2} \mathrm{~S}_{4}$ doped with $3 \%$ In. The In-doping is supposed to create $\mathrm{In}^{3+}$ defect states and to introduce additional charge carriers to the system. Correspondingly the conductivity as shown in Fig. 5b at room temperature is enhanced by about one decade compared to the nominally undoped $\mathrm{CdCr}_{2} \mathrm{~S}_{4}$ (Fig. 3). However, due to the use of chlo- 


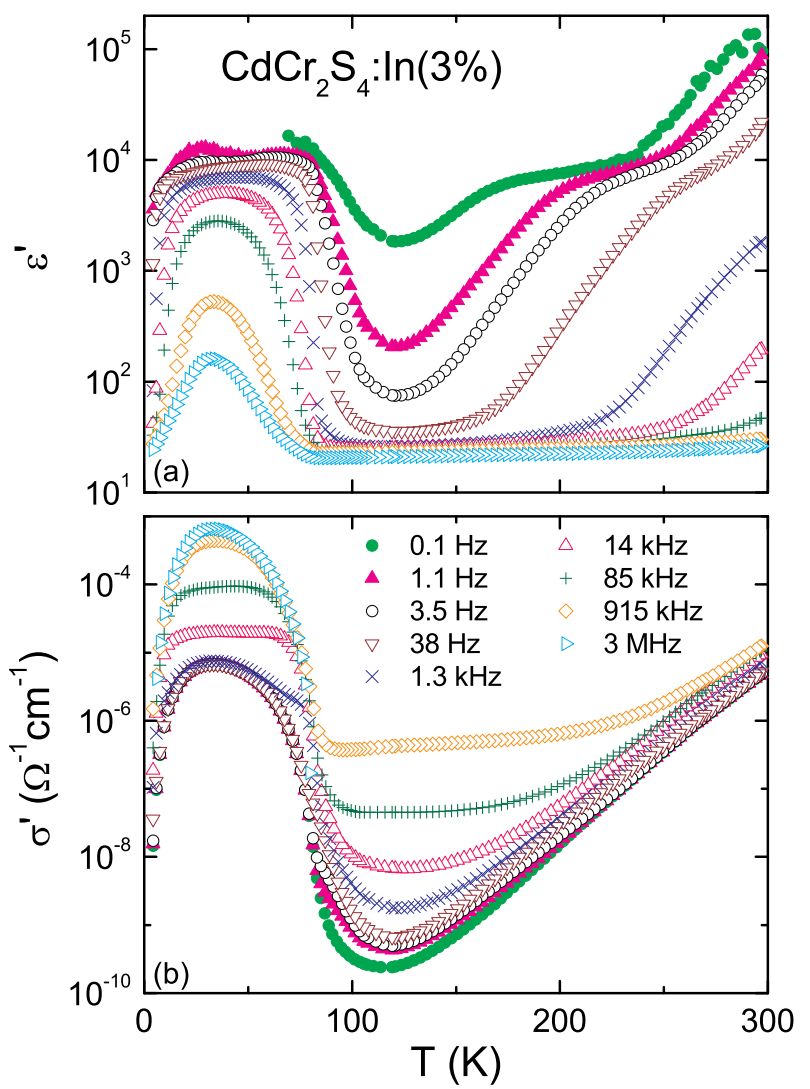

FIG. 5: Temperature dependence of the real part of the dielectric permittivity $\varepsilon^{\prime}$ (upper frame) and the ac-conductivity $\sigma^{\prime} \propto \omega \varepsilon^{\prime \prime}$ (lower frame) of $\mathrm{CdCr}_{2} \mathrm{~S}_{4}$ doped with $3 \%$ In for various frequencies.

rine as transport agent for the crystal growth, even in the pure compound a finite charge doping in the subpercent range has to be considered. The general behavior of the In-doped compound is to be similar to that of the undoped one. Below the magnetic transition at roughly $80 \mathrm{~K}$ a pronounced increase of both quantities, $\varepsilon^{\prime}$ and $\sigma^{\prime}$, is detected. In that temperature range the shape of the broad maximum in the conductivity even displays signs of the discussed two-peak structure, compatible to the re-accelerating and (towards lowest temperatures) re-freezing of the relaxor dynamics. However, the leveling-off in $\sigma^{\prime}$ above the magnetic transition temperature sets in only for the highest frequencies. In the MHz-range similar values of about $10^{-6} / \Omega \mathrm{cm}$ are measured for the pure and the In-doped compound. This reflects a similar contribution from hopping transport mechanisms showing up in the AC-conductivity. But in $\mathrm{CdCr}_{2} \mathrm{~S}_{4}: \operatorname{In}(3 \%)$ the DC-transport is much more dominating. This hampers the ability to measure dielectric polarization or pyro-currents and to distinguish them from ohmic contributions. As documented in Fig. 5a, the absolute values of the dielectric constant are strongly enhanced compared to pure $\mathrm{CdCr}_{2} \mathrm{~S}_{4}$, reaching step heights

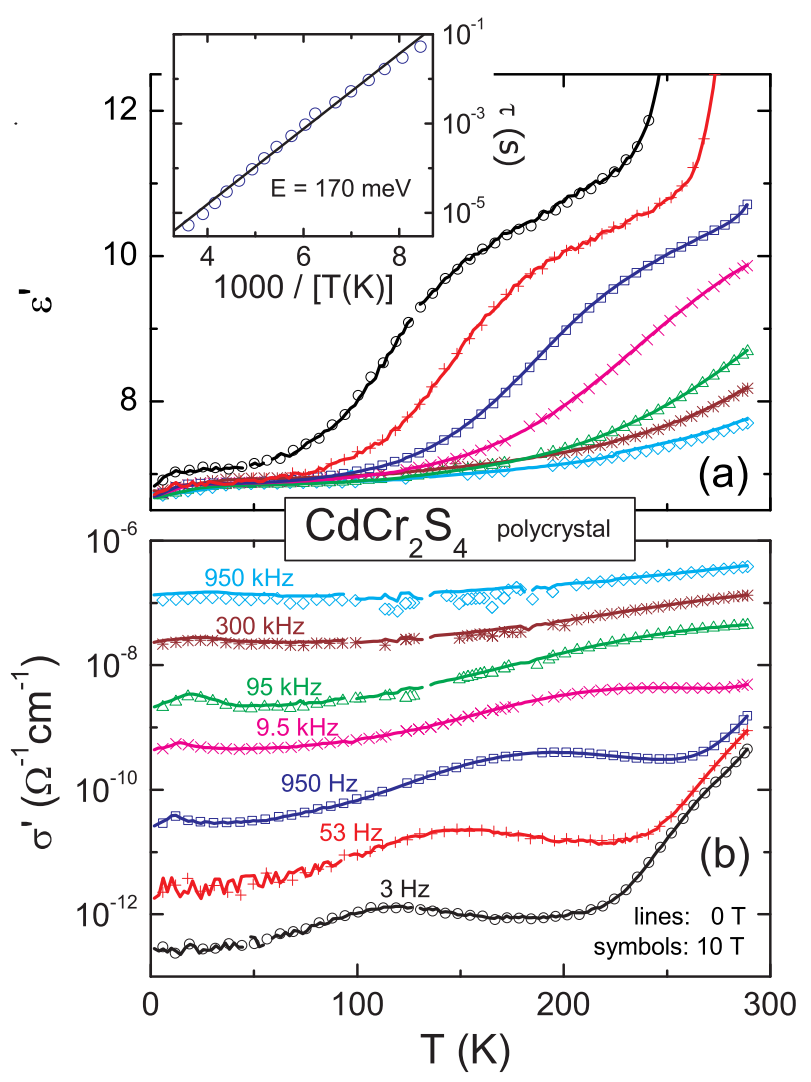

FIG. 6: Temperature dependence of the real part of the dielectric permittivity $\varepsilon^{\prime}$ (upper frame) and the ac-conductivity $\sigma^{\prime} \propto \omega \varepsilon^{\prime \prime}$ (lower frame) of poly-crystalline $\mathrm{CdCr}_{2} \mathrm{~S}_{4}$ for various frequencies.

up to $10^{4}$. At this point it is important to note that the DC-conductivity also exhibits strong changes at the magnetic transition (base on the fact that the $\sigma(T)$ curves for the lowest frequencies in Fig. $5 \mathrm{~b}$ coincide below $80 \mathrm{~K}$, the DC-conductivity in this region is well approximated by the curve at $0.1 \mathrm{~Hz}$ ). However, $\sigma_{D C}$ does of cause not have any influence on the dielectric constant. This is in contrast to the discussed AC-contributions of the conductivity which via the Kramers-Kronig relation has to appear in $\sigma^{\prime}$ and $\sigma^{\prime \prime}$ and thus also leads to a contribution to the dielectric constant $\varepsilon^{\prime} \propto \sigma^{\prime \prime} / \omega .^{28}$ However, the steps in the dielectric constant, discussed already for pure $\mathrm{CdCr}_{2} \mathrm{~S}_{4}$ in the paramagnetic regime still can be detected. The relaxational character of this contribution, together with the fact that the AC-conductivity seems to be of similar magnitude for the doped and the undoped compound, points against an origin related to incoherent transport processes. The thermal activation of this relaxational process in $\mathrm{CdCr}_{2} \mathrm{~S}_{4}: \operatorname{In}(3 \%)$ can be characterized by $E_{B} \approx 200 \mathrm{meV}$ and $\tau_{0} \approx 10^{-7} \mathrm{~s}$. The latter value is considerably higher than in nominally undoped $\mathrm{CdCr}_{2} \mathrm{~S}_{4}$. This could be interpreted with larger relaxing clusters, which also would be compatible with the enhanced values of the permittivity. 
However, towards higher temperatures an increasing background, like in $\mathrm{CdCr}_{2} \mathrm{~S}_{4}$, comes into play. This contribution may indeed arise from non-intrinsic, so-called Maxwell-Wagner type, contributions. ${ }^{29,30}$ At the sample surfaces the generation of a Schottky-type of diode between the metallic electrode and the semiconducting bulk material can give rise to a depletion layer, possessing high resistance and capacitance. Both can be modeled by a parallel RC-element connected in series with the bulk sample, leading to a characteristic relaxation time, which changes due to the temperature dependence of the conductivity. In this case the observed relaxation feature would not be a bulk property. Prominent examples for Maxwell-Wagner relaxations with very high (typically $10^{3}-10^{5}$ ), non-intrinsic values for $\varepsilon^{\prime}$ are the socalled "colossal dielectric constant" materials ${ }^{29}$. Such effects may additionally influence the increase of $\varepsilon^{\prime}(T)$ above the discussed intrinsic relaxation step. A possibility to distinguish between both scenarios is the variation of the electrode material by using contacts made from silver-paint or sputtered gold. In both cases discussed so far, namely nominally pure single-crystalline $\mathrm{CdCr}_{2} \mathrm{~S}_{4}$ (Fig. 3) and $\mathrm{CdCr}_{2} \mathrm{~S}_{4}: \operatorname{In}(3 \%)$ (Fig. 5) significant differences between the results obtained for different electrode materials could only be detected for the non-intrinsic high-temperature wing of the permittivity, showing up, e.g., in Fig. $5 \mathrm{a}$ at $T>240 \mathrm{~K}$ at $\nu=0.1 \mathrm{~Hz}$. In contrast, as demonstrated for the pure compound in [23], the relaxation features discussed above are still observed at the same temperatures with the same amplitude.

An alternative approach is to regard stoichiometric but poly-crystalline $\mathrm{CdCr}_{2} \mathrm{~S}_{4}$. Fig. 6 represents plots of $\varepsilon^{\prime}(T)$ and $\sigma^{\prime}(T)$ for pressed powder samples. Here, in contrast to the $\mathrm{CdCr}_{2} \mathrm{~S}_{4}$ single crystals, traces of $\mathrm{Cl}$ and corresponding impurity charge doping can not be excludd. Accordingly, the conductivity is much reduced as displayed in Fig. 6b. The complete temperature range is dominated by incoherent AC-conductivity, and only for the lowest measured frequencies, in the Hz-range, the characteristic increase with increasing temperature typical for thermally activated DC-transport, can be observed. Compared to the single crystalline sample (Fig. 3b), the AC-conductivity is reduced by less than one decade only, while, e.g. at room temperature, the DC-conductivity is about three decades smaller in the poly-crystal. Also the dielectric response in the poly-crystal is much weaker. Nevertheless, a relaxational step in $\varepsilon^{\prime}(T)$ for temperatures above $100 \mathrm{~K}$ can be observed. The thermal activation of this relaxation process (see inset of Fig. 6) in poly-crystalline $\mathrm{CdCr}_{2} \mathrm{~S}_{4}$ can roughly be characterized with an activation barrier of about $E_{B} \approx 170 \mathrm{meV}$ and $\tau_{0} \approx 6 \times 10^{-9} \mathrm{~s}$, both values being compatible with the parameter range of the single crystals discussed above. Thus this much smaller dielectric contribution could be interpreted as a remainder of the pronounced single-crystal phenomena. However, the strong anomaly at the magnetic transition is completely absent in the poly-crystal. In addition, no magnetic field dependence

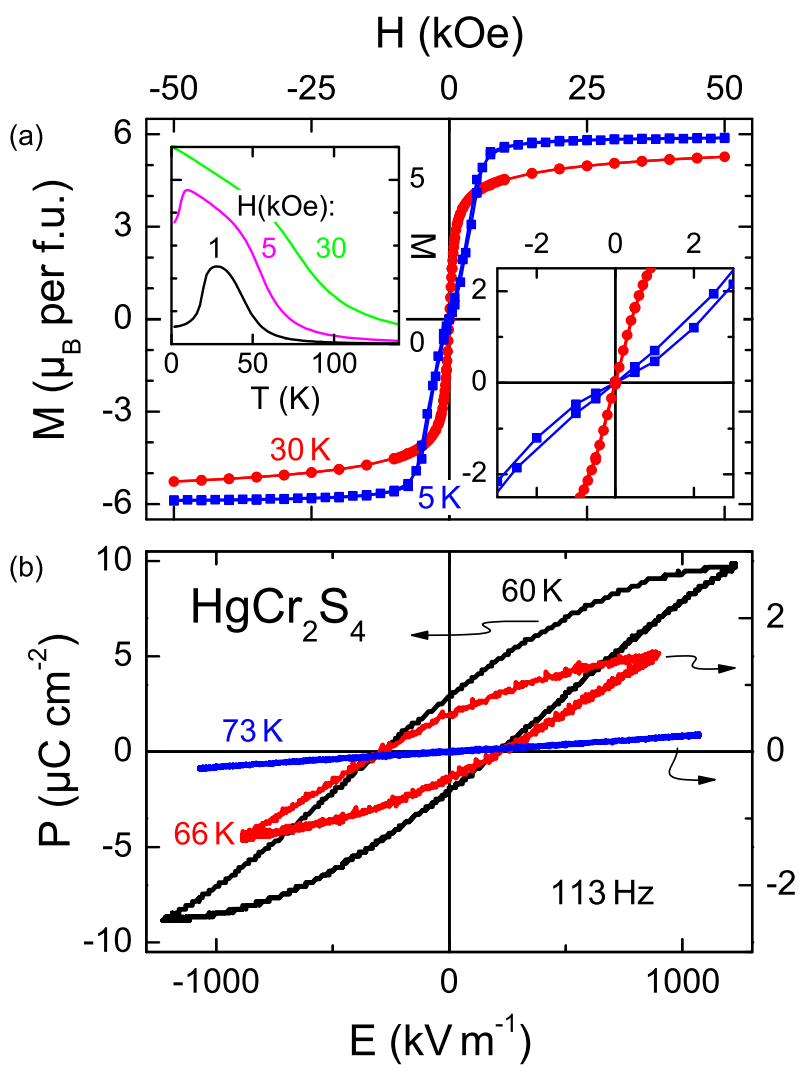

FIG. 7: Hysteresis loops for the magnetic $(M(H),(\mathrm{a}))$ and dielectric sector $(P(E)$, (b)) measured at various temperatures for $\mathrm{HgCr}_{2} \mathrm{~S}_{4}$. The polarization data was measured at $\nu=113 \mathrm{~Hz}$ ([12]; copyright (2005) by the APS). The insets show the temperature dependence of the magnetization for various fields and a magnification of the magnetic hysteresis loops for low fields.

can be found in the vicinity of the magnetic transition, which still occurs at about $84 \mathrm{~K}$ (compare the solid lines $(0 \mathrm{~T})$ and the symbols $(10 \mathrm{~T})$ in Fig. 6). This seems to point to the necessity of additional charge carriers and/or defect states for the microscopic coupling mechanism between dielectric and magnetic polarization. On the other hand, the absence of inner strains and the possible variation of stoichiometry at the grain boundaries could play an important role for the suppression of the magneto-capacitive effects in the poly-crystalline material. In this context it is interesting that annealing of the single-crystalline $\mathrm{CdCr}_{2} \mathrm{~S}_{4}$ samples, both, in vacuum or sulphur atmosphere, leads to a suppression of the frequency dependent relaxation features above and below $T_{c}$ and no remanent electric polarization can be found at low temperatures. Further systematic studies of this issue are highly needed and currently being performed. 

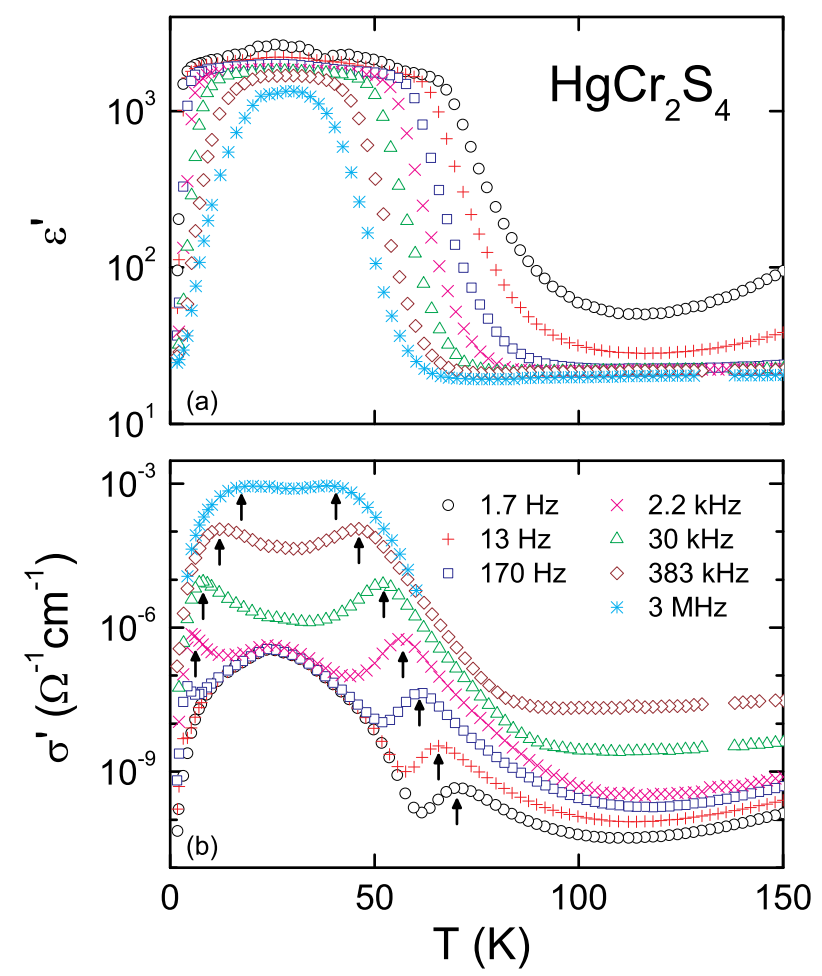

FIG. 8: Temperature dependence of real part of the dielectric permittivity $\varepsilon^{\prime}(\mathrm{a})$ and the ac-conductivity $\sigma^{\prime} \propto \omega \varepsilon^{\prime \prime}(\mathrm{b})$ of $\mathrm{HgCr}_{2} \mathrm{~S}_{4}$ for various frequencies ([12]; copyright (2005) by the APS).

\section{C. $\mathrm{HgCr}_{2} \mathrm{~S}_{4}$}

Even though the observed magneto-capacitive effects are relatively sensitive to the details of the sample preparation and impurity concentration, they seem to be stable against the exchange of the chalcogen ion. Replacing sulphur by selenium leads to $\mathrm{CdCr}_{2} \mathrm{Se}_{4}$, in which the ferromagnetic transition is increased towards $T_{c} \approx 125 \mathrm{~K} .^{23}$ At this temperature a pronounced variation of $\varepsilon^{\prime}(T)$ can be found denoting a similarly strong magneto-dielectric coupling like in $\mathrm{CdCr}_{2} \mathrm{~S}_{4}$. Also the typical relaxor peaks in the real part of the permittivity at $T>T_{c}$ are shifted to higher temperatures in $\mathrm{CdCr}_{2} \mathrm{Se}_{4}$. In the following we discuss the compound $\mathrm{HgCr}_{2} \mathrm{~S}_{4}$ where instead of the chalcogen the $A$-site ion is replaced. The different ionic size in the first instance changes the bond-distances of the spinel structure, which, nevertheless, stays cubic at room temperature. ${ }^{20}$ This induces a redistribution of the interaction strengths between nearest and next-nearest super-exchange as well as direct $B-B$ exchange pathes. This results in a bond-frustrated scenario with complex magnetic properties. Below $T_{N}=22 \mathrm{~K}$ a helical antiferromagnetic structure is established in the absence of a magnetic field. However, already in small external magnetic fields ferromagnetic order can be induced. Fig. 7a shows magnetic hysteresis measurements for $T=5$ and

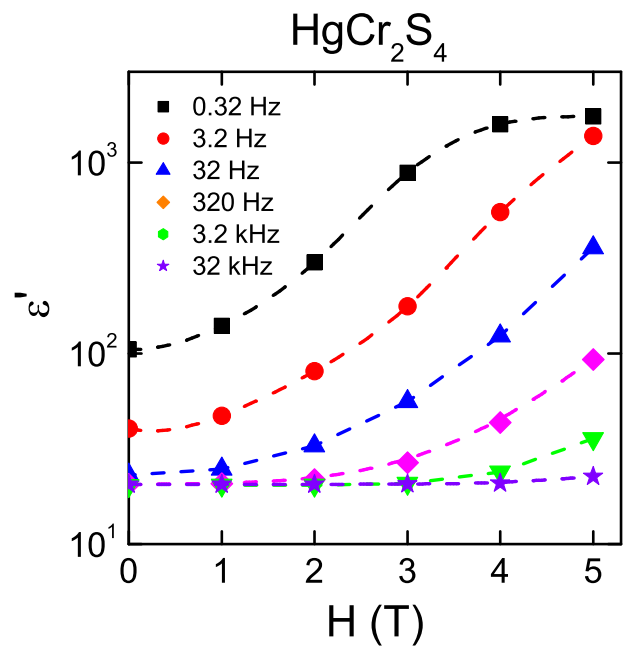

FIG. 9: Magnetic field dependence of $\varepsilon^{\prime}$ in $\mathrm{HgCr}_{2} \mathrm{~S}_{4}$ for various frequencies at $T=120 \mathrm{~K}$.

$30 \mathrm{~K}$. The slight S-shape of the low-temperature curve, observed at small fields, denotes the metamagnetic transition from antiferromagnetic into induced ferromagnetic order reaching the full expected ordered moment of $6 \mu_{B}$ $\left(3 \mu_{B}\right.$ per $\left.\mathrm{Cr}^{3+}\right)$ in fields above $1 \mathrm{~T}$. Even though at temperatures above $T_{N}$ no spontaneous ferromagnetic order can be detected (as monitored in the upper inset of Fig. 7 via the $M(T)$ curve measured at $1 \mathrm{kOe}$ ) in fields even below $1 \mathrm{~T}$ a large ferromagnetic component can be induced. This denotes the presence of strong ferromagnetic fluctuations and the vicinity of the system to ferromagnetic order. ${ }^{31}$ At the same time (Fig. 7b) a dielectric polarization hysteresis appears for temperatures below $70 \mathrm{~K}$. The values for the remanent polarization in $\mathrm{HgCr}_{2} \mathrm{~S}_{4}$ are even higher than in $\mathrm{CdCr}_{2} \mathrm{~S}_{4}$. Due to the finite conductivity in $\mathrm{HgCr}_{2} \mathrm{~S}_{4}$ (shown below), the interpretation of such type of dielectric hysteresis measurements is not completely unambiguous. In ref. 32 possible reasons for non-intrinsic non-linearities of the dielectric response are discussed. One indication for extrinsic behavior could be the frequency dependence, which is present in the case of $\mathrm{HgCr}_{2} \mathrm{~S}_{4}$. This criterion is, however, of course also compatible with the picture of a relaxor ferrolectric state. A second criterion would be the vanishing of a saturation for increasing frequencies, which is in contrast to the experimental findings. ${ }^{12}$ An alternative determination of the polarization via measurements of the pyro-current unfortunately fails due to the relative high conductivity of the samples. In addition, the variation of the electrode material and the investigation of different sample batches yield similar dielectric properties, which points against an extrinsic nature of the observed phenomena. ${ }^{12}$

Fig. 8 displays the temperature dependence of the real parts of dielectric constant and conductivity for various frequencies in the temperature range below $150 \mathrm{~K}$. The response at higher temperatures is dominated by extrin- 

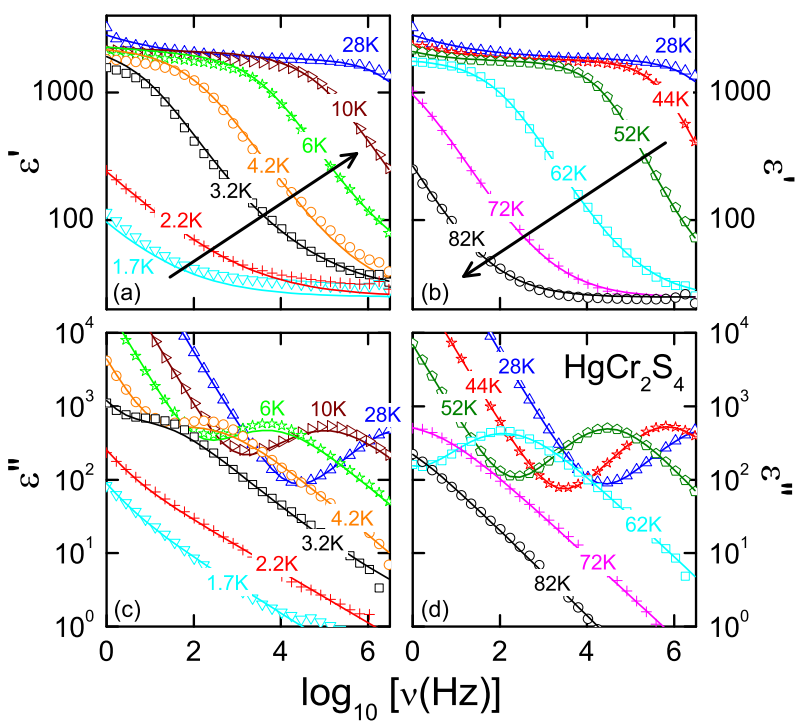

FIG. 10: Frequency dependence of the complex dielectric permittivity $\varepsilon^{*}((\mathrm{a}, \mathrm{b})$ : real part; $(\mathrm{c}, \mathrm{d})$ : loss) as measured for various temperatures in $\mathrm{HgCr}_{2} \mathrm{~S}_{4}$. The arrows indicate the shift of the mean relaxation rate $\nu_{0}=1 / 2 \pi \tau_{0}$ with temperature.

sic Maxwell-Wagner type contributions as indicated by the dependency of the the data on the choice of electrode material. ${ }^{12}$ The results of Fig. 8 qualitatively resemble those of the findings in $\mathrm{CdCr}_{2} \mathrm{~S}_{4}$ and $\mathrm{CdCr}_{2} \mathrm{~S}_{4}: \operatorname{In}(3 \%)$ in the corresponding temperature regime. The conductivity is significantly higher than in $\mathrm{CdCr}_{2} \mathrm{~S}_{4}$. A double peak structure in $\sigma^{\prime}(T)$ at $T<75 \mathrm{~K}$ can clearly be resolved. This behavior again can be explained by the re-acceleration of relaxor entities due to the presence of strong ferromagnetic correlations and the subsequent refreezing towards lower temperatures. The peaks in the conductivity coincide with the points of inflection in the dielectric constant (Fig. 8a). The plateau-like maximum of $\varepsilon^{\prime}$ reaches a value of roughly 2000. The increase of the dielectric constant for temperatures between $40 \mathrm{~K}$ (high frequencies, $3 \mathrm{MHz}$ ) and about $70 \mathrm{~K}$ (low frequencies, $1.7 \mathrm{~Hz}$ ) is much stronger than in $\mathrm{CdCr}_{2} \mathrm{~S}_{4}$. Correspondingly, a pronounced magnetic field dependence of the permittivity can be expected in the regarded temperatures range. Fig. 9 displays the dielectric constant $\varepsilon^{\prime}(H)$ in magnetic fields up to $5 \mathrm{~T}$ for various frequencies. The application of an external magnetic field enhances the ferromagnetic correlations and shifts their appearance towards higher temperatures. As a consequence the mentioned re-acceleration of the dielectric dynamics is induced by the magnetic field and $\varepsilon^{\prime}$ considerably increases with increasing field. This effect is strongly frequency dependent again underlining the relaxational character of the magneto-capacitive coupling. At a temperature of $120 \mathrm{~K}$ and for frequencies in the Hz-regime a magnetic field of $1 \mathrm{~T}$ easily can change the permittivity by a factor of ten. Under the same conditions the response for frequencies above $30 \mathrm{kHz}$ is barely effected. The details of the frequency dependence of the complex permittivity are monitored in Fig. 10. The representation as permittivity spectra $\varepsilon^{*}(\omega)$ contains the most significant information on the relaxation dynamics. The condition $\tau=1 / 2 \pi \nu$ where $\nu$ is the position of the maximum in the dielectric loss or the point of inflection in the step of the dielectric constant allows for the straightforward determination of the relaxation time $\tau$. Typical relaxation steps in $\varepsilon^{\prime}(T)$ are monitored for various temperatures in Figs. 10a and $\mathrm{b}$, and the accompanying loss peaks are observed in $\varepsilon^{\prime \prime}(T)$ in Figs. 10c and d. (Note that the increase in the loss spectra observed towards low frequencies results from finite DC-conductivity as discussed below). Considering the steps in $\varepsilon^{\prime}$ in the low temperature region (Fig. 10a), the usual thermally activated scenario becomes apparent: They shift to higher frequency, i.e. the dynamics becomes faster with increasing temperature. But in the temperature range around the onset of ferromagnetic fluctuations between 30 and $80 \mathrm{~K}$ (Fig. 10b) the opposite behavior is found: The system seems to slow down with increasing temperature! Obviously, in this regime not the temperature but the magnetic correlations seem to determine the relaxation rate. On the other hand the DCconductivity of $\mathrm{HgCr}_{2} \mathrm{~S}_{4}$ experiences very pronounced changes at about $80 \mathrm{~K}$ and also exhibit a strong magnetic field dependence. ${ }^{12,27}$ Thus this material exhibits CMR and CMC properties at the same time. However, a direct relation between both quantities is not obvious, as has been pointed out above, and the relaxational character of the dielectric response does not point towards charge transport as the origin of the strong anomalies in $\varepsilon^{\prime}$. Strong spin-disorder scattering has been postulated for ferromagnetic semiconductors with very small charge carrier concentrations. ${ }^{33}$ An enhanced local electronic polarizability could link the charge transport to the relaxor ferroelectric properties in these slightly doped spinel systems. But further investigations and a more detailed theoretical approach would be necessary to settle such a picture.

A quantitative evaluation of the results shown in Fig. 10 was obtained from the simultaneous fitting of the real and imaginary part of the permittivity data, the resulting fit curves being displayed as solid lines. The fits account for an symmetric (Cole-Cole) distribution of relaxation times centered at $\tau_{0}(T)$, DC-conductivity and DC-dielectric constant, and an AC-conductivity contribution $\sigma_{A C}^{\prime} \propto \nu^{s}$ with $s<1 .{ }^{28}$ The latter term via the Kramers-Kronig relation is related to the imaginary part of the conductivity and thus also gives a contribution to the real part of the permittivity $\varepsilon^{\prime} \propto \sigma^{\prime \prime} / \omega$. In literature this power-law type of contribution is referred to as "universal dielectric response" and usually assumed to account for hopping transport processes. ${ }^{28}$ The mean relaxation times resulting from this type of evaluation are displayed as $\tau(1 / T)$ in Fig. 11 for $\mathrm{HgCr}_{2} \mathrm{~S}_{4}$ and $\mathrm{CdCr}_{2} \mathrm{~S}_{4}$. At high temperatures (small values of $1 / T$; see inset of Fig. 11) the mentioned conventional relaxational freezing scenario, corresponding to an increase 


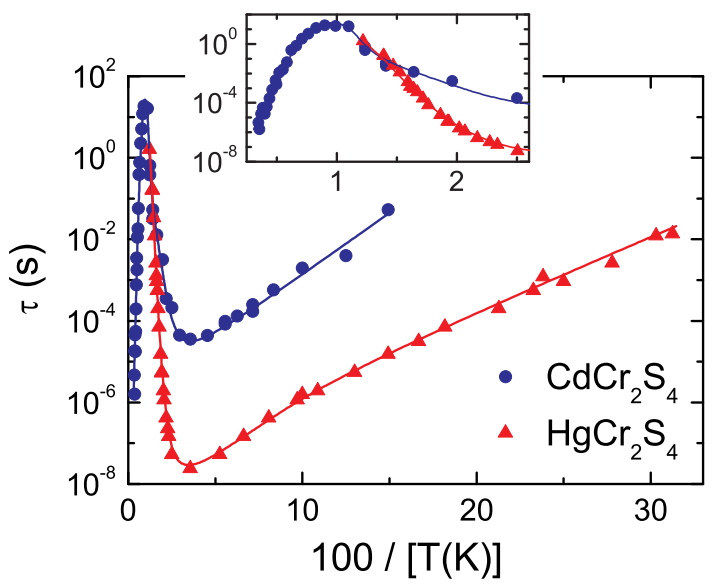

FIG. 11: Comparison of the temperature dependent mean relaxation times of $\mathrm{HgCr}_{2} \mathrm{~S}_{4}$ and $\mathrm{CdCr}_{2} \mathrm{~S}_{4}$ in an Arrheniustype presentation. The inset displays a magnification of the high temperature behavior.

of $\tau$ with decreasing $T$, can be followed for $\mathrm{CdCr}_{2} \mathrm{~S}_{4}$. Then for both compounds an anomalous regime occurs, in which the relaxation times become reduced for decreasing temperatures. In this regime the magnetic field and the magnetization lead to a re-acceleration of the dynamics and the frozen relaxor-system melts again. Finally, at lowest temperatures, where the influence of the magnetization saturates, a scenario of thermal activation becomes dominant again and the dynamics slow down again. In this regime the relaxation time in $\mathrm{HgCr}_{2} \mathrm{~S}_{4}$ is about two decades lower than in $\mathrm{CdCr}_{2} \mathrm{~S}_{4}$. This difference is difficult to explain considering the different magnetic configuration of both compounds. As ferromagnetism seems to decrease $\tau$, the completely ferromagnetically ordered $\mathrm{CdCr}_{2} \mathrm{~S}_{4}$ should rather show the faster dielectric response. The in general larger dielectric response in $\mathrm{HgCr}_{2} \mathrm{~S}_{4}$ also would point towards larger relaxor entities with accordingly higher relaxation times.

\section{CONCLUSIONS AND SUMMARY}

The present dielectric experiments provide clear evidence for an relaxational process using the observed magneto-capacitive phenomena. The ferroelectric distortions are supposed to result from an off-center position of the $\mathrm{Cr}^{3+}$-ions which generates a locally polar but macroscopically isotropic cluster state. ${ }^{11,18}$ Especially in $\mathrm{HgCr}_{2} \mathrm{~S}_{4}$ bond frustration of the magnetic sector may play a considerable role. But the structural frustration within the cubic lattice, which at room temperature is highly symmetric and geometrically unconstrained, is supposed to be the constituting ingredient driving the observed relaxor-like freezing. ${ }^{34}$ However, recent $\mathrm{LDA}+\mathrm{U}$ calculations did not find any indication for the softening of a polar phonon mode which could explain the local ferroelectric distortions. ${ }^{35}$ Nevertheless, pronounced spin-phonon coupling could be investigated via optical spectroscopy in magnetic fields and will be reported in a forthcoming paper. On the other hand, the details of the coupling mechanism between magnetization or the magnetic field and the dielectric permittivity in these compounds is so far unclear. As pointed out above, the relaxation dynamics of the polar moments is accelerated below $T_{c}$, but the microscopic origin of the detected relaxation dynamics and why this dynamics couples so strongly to the magnetic order parameter is still unknown. Most plausible is a coupling via exchange striction, i.e. volume changes arising from the magnetic exchange energy. ${ }^{36}$ The onset of spin order leads to a local magnetostrictive distortion of the lattice. Thus the energy barriers against dipolar reorientation are reduced and the mean relaxation rate is enhanced. An alternative explanation could be a magnetic-field induced variation of charge-carrier mobility or density. As mentioned before, a sizable DC magneto-resistive effect is well known for $\mathrm{CdCr}_{2} \mathrm{~S}_{4}$ and $\mathrm{HgCr}_{2} \mathrm{~S}_{4},{ }^{12,27}$ Even so the DC-conductivity of course not directly contributes to $\varepsilon^{\prime}$, but only to $\varepsilon^{\prime \prime}$. Its variation may denote changes in the electronic polarizability. ${ }^{37,38}$ Within such a scenario one could expect a strong enhancement of the dielectric constant in the vicinity of a metal-insulator transition, e.g. driven by charge doping of semiconductors. ${ }^{39,40}$ However, the influence of purely electronic degrees of freedom probably can not explain the observed dispersion in the investigated frequency range. Finally, even though the influence of the electrode polarization via Maxwell-Wagner type of processes appears to be unlikely if considering our experiments with different types of contacts, the importance of possible internal boundary mechanisms can not be completely ruled out, and needs further clarification. ${ }^{41}$

In summary, our results reveal that in $\mathrm{CdCr}_{2} \mathrm{~S}_{4}$ and $\mathrm{HgCr}_{2} \mathrm{~S}_{4}$ ferromagnetic order, or at least strong ferromagnetic fluctuations, coexists with a relaxorferroelectric state, characterized by a significant relaxational behavior. Both properties are strongly coupled. The observed strong increase of the dielectric permittivity in the magnetically ordered state of these compounds can be explained by a re-acceleration of the dielectric relaxor dynamics. These are influenced by the onset of magnetization, which can be driven by external magnetic fields. This results in a colossal magneto-capacitive effect, which exhibits a pronounced frequency dependence. Concerning the microscopic origin of the observed effects further investigations are needed.

\section{Acknowledgments}

This work was partly supported by the Bundesministerium für Bildung und Forschung (BMBF) via Grant No. VDI/EKM 13N6917-A and by the Deutsche Forschungsgemeinschaft via Sonderforschungsbereich SFB 484 (Augsburg). 
1 M. Fiebig, J. Phys. D: Appl. Phys. 38, R123 (2005).

2 J. Valasek, Phys. Rev. 17, 475 (1921).

3 A. von Hippel, Rev. Mod. Phys. 22, 221 (1950).

4 E. Ascher, H. Rieder, H. Schmid, and H. Stössel, Phys. Rev. B 37, 1404 (1966).

5 T. Goto, T. Kimura, G. Lawes, A. P. Ramirez, and Y. Tokura, Phys. Rev. Lett. 92, 257201 (2004).

6 T. Kimura, T. Goto, H. Shintani, K. Ishizaka, T. Arima, and Y. Tokura, Nature 426, 55 (2003).

7 N. Hur, S. Park, P. A. Sharma, J. S. Ahn, S. Guha, and S. W. Cheong, Nature 429, 392 (2004).

8 T. Lottermoser, T. Lonkai, U. Amann, D. Hohlwein, J. Ihringer, and M. Fiebig, Nature 430, 514 (2004).

9 M. Fiebig, T. Lottermoser, D. Frohlich, A. V. Goitsev, and R. V. Pisarev, Nature 419, 818 (2002).

10 G. Lawes, A. B. Harris, T. Kimura, N. Rogado, R. J. Cava, A. Aharony, O. Entin-Wohlman, T. Yildirim, M. Kenzelmann, C. Broholm, et al., Phys. Rev. Lett. 95, 087205 (2005).

11 J. Hemberger, P. Lunkenheimer, R. Fichtl, H.-A. Krug von Nidda, V. Tsurkan, and A. Loidl, Nature 434, 364 (2005).

12 S. Weber, P. Lunkenheimer, R. Fichtl, J. Hemberger, V. Tsurkan, and A. Loidl, Phys. Rev. Lett. 96, 157202 (2006).

13 J. E. W. Verwey, Nature 144, 327 (1939).

${ }^{14}$ V. Fritsch, J. Hemberger, N. Büttgen, E. W. Scheidt, H.A. Krug von Nidda, A. Loidl, and V. Tsurkan, Phys. Rev. Lett. 92, 116401 (2004).

15 R. Fichtl, V. Tsurkan, P. Lunkenheimer, J. Hemberger, V. Fritsch, H.-A. Krug von Nidda, E.-W. Scheidt, and A. Loidl, Phys. Rev. Lett. 94, 027601 (2005).

16 M. Brando, R. Caciuffo, J. Hemberger, A. Loidl, L. Malavasi, and P. Ghigna, New Journal of Physics 6, 191 (2004).

17 N. A. Hill, J. Phys. Chem. B 104, 6694 (2000).

18 N. Grimes, Phil. Mag. 26, 1217 (1972).

19 H. Göbel, J. Magn. Magn. Mater. 3, 143 (1976).

${ }^{20}$ V. Tsurkan, J. Hemberger, A. Krimmel, H.-A. Krug von Nidda, P. Lunkenheimer, S. Weber, V. Zestrea, and A. Loidl (cond-mat/0603348).

21 U. S. et al., Ferroelectrics 249, 89 (2001).

22 J. Hemberger, Dielectric spectroscopy at the orientationalglass transition (Shaker, Aachen, 1997).

23 J. Hemberger, P. Lunkenheimer, R. Fichtl, S. Weber, V. Tsurkan, and A. Loidl (Proccedings of SCES05 (in press), cond-mat/0508014).

24 L. Cross, Ferroelectrics 76, 241 (1987).

25 P. Lunkenheimer, R. Fichtl, J. Hemberger, V. Tsurkan, and A. Loidl, Phys. Rev. B 72, 060103(R) (2005).

26 A. Levstik, Z. Kutnjak, C. Filipič, and R. Pirc, Phys. Rev. B 57, 11204 (1998).

27 H. Lehmann and M. Robbins, J. Appl. Phys. 37, 1389 (1966).

28 A. K. Jonscher, Dielectric Relaxations in Solids (Chelsea Dielectrics Press, London, 1983).

29 P. Lunkenheimer, R. Fichtl, S. G. Ebbinghaus, and A. Loidl, Phys. Rev. B 70, 172102 (2004).

30 R. Wagner, Science 40, 817 (1913).

31 H. W. Lehmann and G. Habeke, Phys. Rev. B 1, 319 (1970).

32 L. Pintilie and A. Alexe, Appl. Phys. Lett. 87, 112903 (2005).

33 P. Majumdarand and P. B. Littlewood, Nature 395, 479 (1998).

34 A. Ramirez, Nature 421, 483 (2003).

${ }^{35}$ C. J. Fennie and K. Rabe, Phys. Rev. B 72, 214123 (2005).

36 G. Martin, A. T. Kellogg, R. L. White, and R. M. White, J. Appl. Phys. 40, 1015 (1969).

37 P. Monceau, F. Y. Nad, and S. Brazovskii, Phys. Rev. Lett. 86, 4080 (2001).

38 D. Staresinic, P. Lunkenheimer, J. Hemberger, K. Biljakovic, and A. Loidl, Phys. Rev. Lett. 96, 046402 (2006).

39 T. G. Castner, Phil. Mag. B 42, 873 (1980).

40 T. G. Aebischer, D. Baeriswyl, and R. M. Noack, Phys. Rev. Lett. 86, 468 (2001).

41 M. Toda, Proceedings of the 3rd Conference on Solid State Devices, Tokyo, 1971 (Japanese Society of Applied Physics, Tokyo, 1972), vol. 41, p. 183. 